\title{
Current status of simulation training in plastic surgery residency programs: A review
}

\author{
Jennifer E. Thomson, Grace Poudrier, John T. Stranix, Catherine C. Motosko, Alexes Hazen \\ Hansjörg Wyss Department of Plastic Surgery, NYU Langone Health, New York, NY, USA
}

Increased emphasis on competency-based learning modules and widespread departure from traditional models of Halstedian apprenticeship have made surgical simulation an increasingly appealing component of medical education. Surgical simulators are available in numerous modalities, including virtual, synthetic, animal, and non-living models. The ideal surgical simulator would facilitate the acquisition and refinement of surgical skills prior to clinical application, by mimicking the size, color, texture, recoil, and environment of the operating room. Simulation training has proven helpful for advancing specific surgical skills and techniques, aiding in early and late resident learning curves. In this review, the current applications and potential benefits of incorporating simulation-based surgical training into residency curriculum are explored in depth, specifically in the context of plastic surgery. Despite the prevalence of simulation-based training models, there is a paucity of research on integration into resident programs. Current curriculums emphasize the ability to identify anatomical landmarks and procedural steps through virtual simulation. Although transfer of these skills to the operating room is promising, careful attention must be paid to mastery versus memorization. In the authors' opinions, curriculums should involve step-wise employment of diverse models in different stages of training to assess milestones. To date, the simulation of tactile experience that is reminiscent of real-time clinical scenarios remains challenging, and a sophisticated model has yet to be established.

Keywords Simulation training / Education, medical / Surgery, plastic / Internship and residency
Correspondence: Alexes Hazen Hansjörg Wyss Department of Plastic Surgery, NYU Langone Health, 550 1st Avenue, New York, NY 10016, USA

Tel: $+1-212-263-5834$

Fax: +1-212-263-5400

E-mail: Alexes.Hazen@nyumc.org

Received: 27 Oct 2017 - Revised: 1 Nov 2017 • Accepted: 3 Feb 2018

pISSN: 2234-6163 • elSSN: 2234-6171 • https://doi.org/10.5999/aps.2017.01585 • Arch Plast Surg 2018;45:395-402

\section{INTRODUCTION}

An increased emphasis on competency-based learning modules coupled with widespread departure from traditional models of Halstedian apprenticeship have fueled an educational transition away from the "see one, do one, teach one" ideology that is engrained in the culture of medical training. Across many medical specialties, a call for new training methods that facilitate the acquisition of surgical skills outside the operating room (OR) have led many residency programs to incorporate surgical simulation platforms [1-4]. Prior to clinical application, medical trainees can acquire and refine surgical skills in synthetic, virtual, animal, and non-living models. Ideally, a surgical simulator would mimic the size, color, texture, recoil and environment of the $\mathrm{OR}[1,5]$, but to date, simulation technology is not yet capable of accurately replicating the physical attributes of human anatomy. Irrespective of these shortcomings, the value of simulation-based training is widely recognized with improvements in 
resident competency scores and surgical performance in the $\mathrm{OR}$ in both early and late stages of residency training [1,5-15].

Despite vast potential for use in residency programs nationwide, current application of simulation-based training in plastic surgery appears extremely limited [16]. In a 2014 survey, utilization of surgical simulation by plastic surgery residents on a monthly basis was reported by only five of the 44 programs surveyed [17]. Although $80 \%$ of programs agreed that simulation based training should be mandatory, only $40 \%$ offered the opportunity for evaluation of trainee teaching sessions, or for formal course completion prior to clinical application [18]. Similarly, a 2016 survey of 89 microsurgery programs revealed that, clinical observation was disproportionately the most common method of training, implemented at a rate of $72 \%$, compared with small animal models (67\%), training courses or videos (33\%), cadavers $(28 \%)$, and high fidelity microsurgery simulators $(2 \%)$.

Given its success in other surgical specialties, the demand for simulation-based training in plastic surgery residency programs is certain to increase $[10-12,15,17,19-21]$. Further research is needed to determine how to best implement and improve the quality of simulation-based surgical training into the plastic surgery residency curriculum. In this review, current applications and potential benefits of incorporating simulation-based surgical training into a plastic surgery residency curriculum are explored in depth.

\section{METHODS}

An electronic literature search of the MEDLINE (PubMed) and Cochrane databases was performed using search terms simulation-based surgical training, technology for surgical education, and plastic surgery and simulation training. Studies were included if they (1) evaluated simulation-based models in improving resident competency; (2) described the application of simulation-based models in the field of plastic surgery; (3) published between January 2000 and June 2016; and (4) were available in English.

Initial search yielded 8,197 articles. The search was then narrowed to include studies published from January 2000 through June 2015. Of the 5,666 articles, 292 articles were written in a language other than English and excluded from the study. The titles and abstracts of the remaining 5,374 studies were subsequently appraised to identify eligible articles. Thirteen papers met inclusion criteria (Table 1) [22-33]. Simulation models used in plastic surgery were evaluated for cost, effectiveness, and realism.

Table 1. Simulation platforms currently implemented in plastic surgery

\begin{tabular}{|c|c|c|c|}
\hline Author (year) & & Platform & Principle evaluation \\
\hline \multicolumn{4}{|l|}{ Computer-based } \\
\hline Schendel et al. 2005 [22] & Craniofacial & $\begin{array}{l}\text { High resolution meshes used to simulate bone and soft } \\
\text { tissue for the repair of cleft lips }\end{array}$ & Identification of anatomical landmarks \\
\hline Stern et al. 2012 [23] & Breast reconstruction & $\begin{array}{l}\text { Breast reconstruction post mastectomy using 3D computer } \\
\text { simulation }\end{array}$ & $\begin{array}{l}\text { Anatomical identification, procedural knowledge, indication } \\
\text { and complication }\end{array}$ \\
\hline Oliker et al. 2012 [24] & General reconstruction & $\begin{array}{l}\text { Computer software replication anatomy, soft tissue } \\
\text { deformities and surgical tool maneuvers }\end{array}$ & $\begin{array}{l}\text { Anatomical identification, procedural knowledge, } \\
\text { identification of surgical tools }\end{array}$ \\
\hline Linke et al. 2013 [25] & NA & Computer based temporal bone surgery & NA \\
\hline Mitchell et al. 2016 [26] & Microsurgery & $\begin{array}{l}\text { Local flap 3D simulator using mass-spring design on a } \\
\text { computer platform }\end{array}$ & Ability to perform procedure \\
\hline \multicolumn{4}{|l|}{ Synthetic } \\
\hline Zheng et al. 2015 [27] & Craniofacial & $\begin{array}{l}\text { Wax model to aid in development of psychomotor and } \\
\text { manual skills involved in cheiloplasty }\end{array}$ & Physical attributes, ability to perform tasks \\
\hline Kazan et al. 2016 [28] & Breast reconstruction & $\begin{array}{l}\text { Silicone and wax model mimicking anatomical appearance } \\
\text { and texture of breast and chest wall for mammoplasty } \\
\text { simulation }\end{array}$ & $\begin{array}{l}\text { Ability to perform tasks, value, relevance, physical } \\
\text { attributes, realism, experience }\end{array}$ \\
\hline \multicolumn{4}{|l|}{ Animal } \\
\hline Lausada et al. 2005 [29] & Microsurgery & Cryopreserved rat aortas for anastomosis & Ability to perform procedure \\
\hline Ghanem et al. 2016 [30] & Microsurgery & Cryopreserved rat aortas for anastomosis practice & Ten common errors \\
\hline \multicolumn{4}{|c|}{ Cadaver } \\
\hline Kligman et al. 2010 [31] & Microsurgery & Use excised tissue to practice anastomosis & Ability to perform task \\
\hline Carey et al. 2014 [32] & General reconstruction & Perfused cadaver for practice of various procedures & Ability to perform procedure \\
\hline Sheckter et al. 2013 [33] & General reconstruction & Cadaver use for procedural practice & $\begin{array}{l}\text { Anatomical dissection and various surgical skills based on } \\
\text { confidence levels }\end{array}$ \\
\hline \multicolumn{4}{|c|}{$\begin{array}{l}\text { Platforms were categorized based on primary method of simulation. Evaluation to verify improvement through simulator use was identified in addition to the broad areas of } \\
\text { plastic surgery which the simulators were applied in. } \\
\text { 3D, three-dimensional; NA, not available. }\end{array}$} \\
\hline
\end{tabular}




\section{RESULTS}

\section{Virtual simulations}

Unique to virtual simulators is the capacity to replicate rare clinical cases that surgeons would not otherwise encountered [5]. Furthermore, although the initial cost of program set up can be high—approximately $\$ 2,000$ per simulator-they facilitate repetition without replacement of materials, which is cost-effective in the long run $[16,34]$. The potential for repetition has led opponents of virtual based learning modules to critique the potential for memorization as opposed to the active learning of new skill sets. Similarly, others maintain that the anatomical variation observable across patient populations cannot be adequately represented through simulators, and that the experience of physical resistance or recoil encountered in live surgery is impossible to accurately recreate $[22,25]$.

Research conducted by Stern et al. [23] utilized virtual simulations to assess resident competency in the identification of complex anatomical landmarks and sequential latissimus dorsi flap reconstruction procedural steps. Residents were evaluated for their ability to correctly identify anatomy and answer a series of interactive questions. Subsequently, residents received an objective assessment of his or her cognitive competency-in terms of anatomy, procedural knowledge, indications, and complications. The scores of each participant were later stratified to indicate relative levels of surgical experience and knowledge of the test material. Pre-test versus post-test evaluation indicated that simulators had effectively improved competency; however, comparison to other simulation models has yet to be investigated [23].

Similarly, Oliker et al. [24] utilized three-dimensional (3D) virtual models to evaluate procedural knowledge for monobloc, Le Fort III, fronto-orbital advancement, and latissimus dorsi flap reconstruction. The interactive questions where designed to gauge resident knowledge of anatomical models, soft tissue deformities, surgical tool motions, chapter divisions, live surgical videos, voiceover audio, and animated transparencies. Although the simulation activity was shown to be beneficial in terms of acquiring and evaluating cognitive surgical knowledge, degree of skill transfer to the OR was not examined [24].

Spring-mass virtual simulators have been developed in an attempt to mimic recoil and tension encountered during surgery. In 2016, Mitchell et al. [26] conducted a local flaps workshop to test the efficacy of mass-spring based simulators for teaching flap designs (e.g., Z-plasty, rhomboid flaps, S-plasty, etc.), allowing participants to perform techniques through a web-based platform. Feedback regarding the simulator was positive for its ability to facilitate step-by-step practice for a given procedure, but the development of a more detailed assessment of resident skill improvement to evaluate its use is needed [35]. The benefits of high resolution meshes resemble those of spring mass models, with the former emulating the physical properties of bone and soft tissue.

Schendel et al. [22] combined high resolution meshes with a spring-mass model for resident training on unilateral cleft lip repair. 3D scans of an 11-day-old patient were translated to a spring model to simulate the effects of recoiling upon incision, as well as meshes, to mimic the physical properties of the anatomical structure involved in the procedure. Six laypersons and six residents were able to rotate and interact in real time, while identifying landmarks for assessment. Each participant interacted four times, with scores improving after each interaction. While participants experienced the simulator as a useful teaching tool, it was incapable of fully mimicking the physical aspects of surgery. The authors note that while improvement in layperson performance was attributable to memorization, the residents were more likely to reflect on their mistakes and to learn new skills with each additional use [22].

Virtual simulation offers the benefit of learning through repetition, at the cost of memorizing material due to lack of variation rather than actively learning as is accomplished from apprentice based training. Applications that require the identification of surgical landmarks or sequential steps can offer residency programs the ability to gauge baseline knowledge prior to clinical application. Notable pitfalls of virtual simulation include the high cost of initial acquisition and the inability to fully simulate the experience of live surgery in an OR setting.

\section{Synthetic models}

The use of synthetic material to simulate tissues and vessels can offer residents an opportunity to practice procedural steps and simultaneously gain experience in the handling and manipulation of surgical tools (Fig. 1) [21]. Common materials, such as silicone and wax, are more affordable than virtual reality [34]. Although the cost of materials is significantly lower than in virtual simulation, synthetic materials must be replaced after each use, and the cost of frequent replacements can add up. Utilization of synthetic materials ranges from practicing sutures to performing full procedures; junior residents are able to learn at their own pace in a safe environment, and subsequently receive objective feedback on their performance [35-37]. A major obstacle in synthetic materials is synthesizing materials that feel and behave as closely as possible to human anatomy. Careful attention to texture, recoil, and anatomy must be present in order for competency to be deemed transferable to the OR [38-41].

In an effort to simulate resident training in mammoplasty, Kazan et al. [28] developed a synthetic Mammoplasty Part-Task 


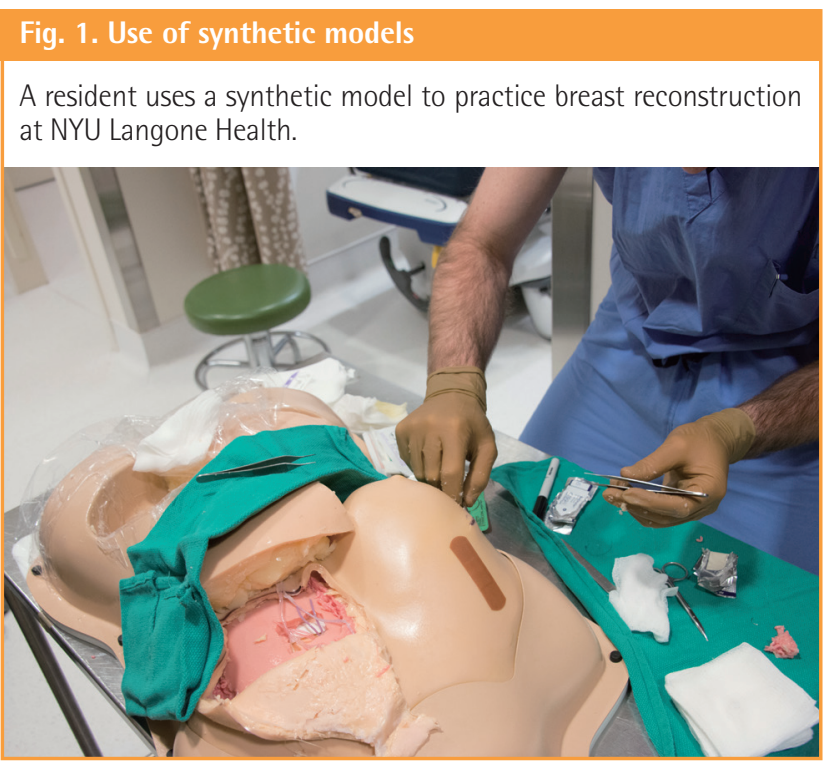

Trainer (MPT). Various layers were created to mimic natural anatomy, including skin, subcutaneous fat, breast tissue, and ribs. Evaluations by four board-certified plastic surgeons were scored on a scale of 0 to 5 and included physical attributes, realism of materials, realism of experience, ability to perform tasks, value, relevance to practice, and overall rating. Although the MPT was able to successfully mimic physical attributes, the realism of materials, especially the subcutaneous fat, was not deemed close enough to live versions [37]. While the model can certainly be used to facilitate procedural learning, its value in terms of transferrable competency remains in question, and realism is lacking.

Synthetic models have been widely used in craniofacial practice $[1,27,37,42,43]$ for both implants and surgical training in complex anatomy. Zheng et al. [27] created a model to aid in the development of psychomotor and manual skills involved in cheiloplasty. Wax was utilized followed by silicone to give lifelike texture, hardness, and elasticity [27]. Additionally, a multicomponent cleft palate simulator replicating a 6-month-old's mouth was created by Vadodaria et al [43]. The model included components to replicate the various anatomical aspects involved in the surgery, including mouth, hard palate, and soft palate. Similar to other studies, the models proved helpful in training on surgical techniques but lacked the ability to fully replicate physical tissue [27,43].

Competency in the use of surgical tools is a vital milestone in residency training, with synthetic models allowing for repeated practice. Current models allow for anatomical representation but still lack the ability to fully replicate physical attributes $[27,42,43]$. As a training model, most synthetic materials are far more affordable than virtual simulators, at the price of needing to constantly replace after use [34]. To date, the models made are on a smaller scale and resemble specific anatomical areas. Much research into development of life-like materials is needed, for the closer to real a surgical training is, the more transferable competency will be.

\section{Animal models}

In recent years, there has been a shift away from animal models for surgical training due to ethical concerns regarding their use [1,3-7]. Still, many argue that practice on live or cryopreserved animals provide transferrable skills to the OR. Traditionally, training involved didactic models in which different materials were used (e.g., surgical gloves, surgical gauze, and silastic tubes). However, none of these models compared to rat aortas on account of differences in softness and resistance to the needle [29]. Rat vessels are heavily used in contemporary microsurgery training, specifically for practicing anastomoses $[30,37]$. Many institutions implement 5-day training courses to evaluate residents on their ability to anastomose rat aortas $[30,34]$. Cryopreserved rat aortas are rather inexpensive, easy to obtain, and eliminate ethical issues regarding the use of live animals $[6,7]$. To evaluate resident ability to perform anastomoses and measure transferability to the OR, Ghanem et al. [30] developed the Anastomosis Lapse Index (ALI). The ALI scores anastomoses based on 10 common mistakes: line disruption, partial thickness stitch, unusually large bites causing infoldment, oblique stitch causing tissue distortion, tight sutures causing strangulation of edges, vessel tear, thread in lumen, gap that is more than two ideal spacing, internal valve/large flap, caught back wall or sidewall. Construct validity was carried out by comparing scores between participants of varying skills and experience; validating ALI as a method to evaluate and predict transfer of microsurgical skills to the OR [30].

To measure resident competency prior to clinical application, the development of a simple, valid, and objective low cost assessment method would prove extremely valuable. Use of animal models is not new to surgical training, but the ability to measure skill acquisition and predict the extent to which they are clinically transferable provides additional impetus for their use as a training method [1,3-7]. The cost of training programs is minimized by the use of cryopreserved specimen, and the texture, size, and recoil of vessels is close enough to humans to facilitate realistic experiences. This aspect of realism puts animal models ahead of synthetic or virtual based training; however, ethical concerns regarding the use of animals persist as a major deterrent. Still, animal models remain useful for practicing microsurgical and suturing skills, and cryopreserved models predominate among current residency programs. 


\section{Cadaver and fresh tissue}

Many of the simulation models formerly discussed are limited by their lack of fidelity, which hinders their ability to facilitate surgical competence. Starting in medical school, the use of cadavers is a long standing practice utilized in all levels of medical training $[44,45]$. With human anatomy and physical attributes accurately represented, anatomical variation is also present, thereby challenging trainees to learn and work within these disparities [1-3]. Nonetheless, cadavers still lack physiological perfusion, the simulation of which has been accomplished and proven effective in the context of vascular, neurological, and trauma surgical procedures [32,46-48].

Perfused cadaver utilization in plastic surgery training was explored by Carey et al. [32], in a study that sought to improve the fidelity of cadavers used in microsurgical training. Regional perfusion maintained by a centrifugal circulation was administered directly to proximal vessels of extremity or neck, with full cadaveric pressurization performed via the femoral artery. Arterial pressure could be adjusted based on the procedure at hand, allowing for accurate replication of the physiologic conditions experienced in live surgery. Cutaneous bleeding was confirmed with 11 procedures being taught to residents [32]. Recreation of techniques in an environment which mimics actual surgery has been a long standing feat. By perfusing cadavers to establish physiological conditions, realistic simulation and use of instruments provides the opportunity to train residents on a high fidelity model that facilitates the acquisition of transferrable skills. In a study by Sheckter et al. [33] incorporation of this model into residency training programs resulted in significant increases in confidence levels for all procedures among all resident training levels. Residents were required to attend weekly dissections related to their current rotation, each of which included a lab manager, surgical technician, and attending physician. Although costing over $\$ 53,000$, the curriculum overcame major hurdles in increasing resident confidence over a 5-year trial period. As an alternative to the use of cadavers and as a method to cut costs, many institutions use freshly excised tissue following surgeries such as abdominoplasty and body contouring. This limits practice to specific techniques as opposed to procedures, but can be very useful for teaching competence in surgical tools $[21,37]$. Concern over the use of cadavers is commonplace in regards to their potential to become reservoirs for communicable disease. Despite this fear and the high cost of use, cadavers and fresh tissue models provide the closest replications of live surgery.

\section{DISCUSSION}

The digital age has greatly expanded the potential for techno- logical advancement to enrich residency training programs, but the majority of existing techniques for teaching surgical skills and simulating operative experiences have thus far been developed in surgical specialties outside of plastic surgery. The relative paucity of plastic surgery simulation tools, especially outside of virtual reality programs, may be related to the difficulty in producing soft tissue models that look and feel like the real thing. Unfortunately, realistic replication of tactile surgical experience remains one of the greatest hurdles in simulation training, and a sophisticated synthetic model has yet to be developed.

Compared to the expense of an OR, synthetic models are cost effective and wield the potential to both improve technical skills and effectively replicate relevant surgical anatomy. Design and implementation of synthetic anatomic models for use in plastic surgery training programs has significant potential. While many of the contemporary models discussed focus exclusively on finite technical skills like suturing, large scale, synthetic models can also be developed to evaluate more significant training milestones. This would give residents the opportunity to learn more complex procedures in a controlled environment outside the OR. At NYU Langone Health, we are in the preliminary stages of evaluating a full-scale synthetic model for abdominal free flap breast reconstruction. Initial resident feedback has been positive, and once we have finalized the prototype, we will begin formally incorporating it into our training curriculum in conjunction with virtual simulation programs.

Using our theoretical design as an example, we plan to structure our simulation-based training curriculum in a manner that underscores the importance of correctly identifying anatomical landmarks and procedural steps. Step-wise employment of diverse simulation-based training models (virtual and synthetic) will provide deliberate training [49]. We plan to assess educational milestones across all stages of residency, in accordance with the ACGME Plastic Surgery Milestones [50]. Progress will be measured in terms of technical mastery and improvements in patient care and OR outcomes.

To ensure the acquisition of foundational skills prior to clinical application, our program would begin with pre-tests (e.g., multiple choice questions designed to mimic the architecture of InService Exams) to evaluate baseline knowledge of anatomical structures, tissue planes, key procedures, indications, contraindications and common complications. Next, depending on student training levels, simulators could include a wide range of materials: e.g., in early postgraduate years, synthetic materials may be deployed to refine dexterity and increase student confidence in the manipulation of surgical tools; in later years, residents could proceed to cadaveric simulation of full procedures. Parameters for resident evaluation would be clearly outlined 
and defined, then monitored by faculty instructors. In addition, senior-level residents could be tasked with teaching and evaluating junior-level residents on milestones they have already achieved [51]. Each resident would be evaluated in accordance with the defined milestones, with a passing grade required to advance. Interaction with faculty for questioning and reflection would immediately follow resident interface with the simulators. Furthermore, post-tests (similar in design to the pre-tests) would be administered to evaluate student progress and milestone accomplishments. To ensure mastery, each milestone would culminate with performance evaluations in a live operative setting. We believe that implementation of this tiered learning approach - that is, deliberate simulator training that culminates in a live operative setting - may improve the efficacy of competency-based training in plastic surgery education.

Although the simulation curriculum outlined above is theoretically sound, a number of limitations have precluded its formal adoption and implementation at our own institution. To start, simulation training remains in the developmental phase insofar as few studies have sought to comprehensively compare the efficacy of simulation-based models with that of traditional methods of plastic surgery education. In addition, although synthetic models appear to be promising, they remain expensive, they may not accurately represent the tactile feedback of soft tissue, and there is not yet evidence that they improve knowledge transfer in a cost-effective manner. Equally important, the implementation of simulation-based training curriculum will inevitably require significant time commitments on the part of both residents and faculty, apart from already-busy clinical duties. In order to justify this, robust evidence to demonstrate the utility of simulation technology in plastic surgery training curriculum is necessary. At this time, this evidence does not yet exist.

\section{NOTES}

\section{Conflict of interest}

No potential conflict of interest relevant to this article was reported.

\section{REFERENCES}

1. Lawrence WT. Simulation in plastic surgery training and education: the path forward. Plast Reconstr Surg 2009;123: 739-40.

2. Grober ED, Hamstra SJ, Wanzel KR, et al. Laboratory based training in urological microsurgery with bench model simulators: a randomized controlled trial evaluating the durability of technical skill. J Urol 2004;172:378-81.
3. Sidhu RS, Park J, Brydges R, et al. Laboratory-based vascular anastomosis training: a randomized controlled trial evaluating the effects of bench model fidelity and level of training on skill acquisition. J Vasc Surg 2007;45:343-9.

4. Ghanem AM, Hachach-Haram N, Leung CC, et al. A systematic review of evidence for education and training interventions in microsurgery. Arch Plast Surg 2013;40:312-9.

5. Agha RA, Fowler AJ. The role and validity of surgical simulation. Int Surg 2015;100:350-7.

6. Fonseca AL, Evans LV, Gusberg RJ. Open surgical simulation in residency training: a review of its status and a case for its incorporation. J Surg Educ 2013;70:129-37.

7. Liebert CA, Mazer L, Bereknyei Merrell S, et al. Student perceptions of a simulation-based flipped classroom for the surgery clerkship: a mixed-methods study. Surgery 2016; 160:591-8.

8. Grober ED, Hamstra SJ, Wanzel KR, et al. Validation of novel and objective measures of microsurgical skill: hand-motion analysis and stereoscopic visual acuity. Microsurgery 2003;23:317-22.

9. Chan WY, Matteucci P, Southern SJ. Validation of microsurgical models in microsurgery training and competence: a review. Microsurgery 2007;27:494-9.

10. Guerreschi P, Qassemyar A, Thevenet J, et al. Reducing the number of animals used for microsurgery training programs by using a task-trainer simulator. Lab Anim 2014;48:72-7.

11. Seymour NE, Gallagher AG, Roman SA, et al. Virtual reality training improves operating room performance: results of a randomized, double-blinded study. Ann Surg 2002;236: 458-63.

12. Grantcharov TP, Kristiansen VB, Bendix J, et al. Randomized clinical trial of virtual reality simulation for laparoscopic skills training. Br J Surg 2004;91:146-50.

13. Larsen CR, Soerensen JL, Grantcharov TP, et al. Effect of virtual reality training on laparoscopic surgery: randomised controlled trial. BMJ 2009;338:b1802.

14. Issenberg SB, McGaghie WC, Petrusa ER, et al. Features and uses of high-fidelity medical simulations that lead to effective learning: a BEME systematic review. Med Teach 2005;27:10-28.

15. Fann JI, Caffarelli AD, Georgette G, et al. Improvement in coronary anastomosis with cardiac surgery simulation. J Thorac Cardiovasc Surg 2008; 136:1486-91.

16. Theman TA, Labow BI. Is there bias against simulation in microsurgery training? J Reconstr Microsurg 2016;32:5405.

17. Immenroth M, Burger T, Brenner J, et al. Mental training in surgical education: a randomized controlled trial. Ann Surg 
2007;245:385-91.

18. Al-Bustani S, Halvorson EG. Status of microsurgical simulation training in plastic surgery: a survey of United States program directors. Ann Plast Surg 2016;76:713-6.

19. Black SA, Nestel DF, Kneebone RL, et al. Assessment of surgical competence at carotid endarterectomy under local anaesthesia in a simulated operating theatre. Br J Surg 2010; 97:511-6.

20. Clarke DB, Kureshi N, Hong M, et al. Simulation-based training for burr hole surgery instrument recognition. BMC Med Educ 2016;16:153.

21. da Cruz JS, Dos Reis ST, Cunha Frati RM, et al. Does warmup training in a virtual reality simulator improve surgical performance? A prospective randomized analysis. J Surg Educ 2016;73:974-8.

22. Schendel S, Montgomery K, Sorokin A, et al. A surgical simulator for planning and performing repair of cleft lips. J Craniomaxillofac Surg 2005;33:223-8.

23. Stern C, Oliker A, Napier Z, et al. Integration of surgical simulation in plastic surgery residency training. Stud Health Technol Inform 2012;173:497-9.

24. Oliker A, Napier Z, Deluccia N, et al. Step-based cognitive virtual surgery simulation: an innovative approach to surgical education. Stud Health Technol Inform 2012;173:3257.

25. Linke R, Leichtle A, Sheikh F, et al. Assessment of skills using a virtual reality temporal bone surgery simulator. Acta Otorhinolaryngol Ital 2013;33:273-81.

26. Mitchell NM, Cutting CB, King TW, et al. A real-time local flaps surgical simulator based on advances in computational algorithms for finite element models. Plast Reconstr Surg 2016; 137:445e-452e.

27. Zheng Y, Lu B, Zhang J, et al. CAD/CAM silicone simulator for teaching cheiloplasty: description of the technique. $\mathrm{Br} \mathrm{J}$ Oral Maxillofac Surg 2015;53:194-6.

28. Kazan R, Courteau B, Cyr S, et al. A novel mammoplasty part-task trainer for simulation of breast augmentation: description and evaluation. Simul Healthc 2016;11:60-4.

29. Lausada NR, Escudero E, Lamonega R, et al. Use of cryopreserved rat arteries for microsurgical training. Microsurgery 2005;25:500-1.

30. Ghanem AM, Al Omran Y, Shatta B, et al. Anastomosis Lapse Index (ALI): a validated end product assessment tool for simulation microsurgery training. J Reconstr Microsurg 2016;32:233-41.

31. Kligman BE, Haddock NT, Garfein ES, et al. Microsurgery trainer with quantitative feedback: a novel training tool for microvascular anastomosis and suggested training exercise.
Plast Reconstr Surg 2010:126;328e-330e.

32. Carey JN, Rommer E, Sheckter C, et al. Simulation of plastic surgery and microvascular procedures using perfused fresh human cadavers. J Plast Reconstr Aesthet Surg 2014;67: e42-e48.

33. Sheckter CC, Kane JT, Minneti M, et al. Incorporation of fresh tissue surgical simulation into plastic surgery education: maximizing extraclinical surgical experience. J Surg Educ 2013;70:466-74.

34. Singh M, Ziolkowski N, Ramachandran S, et al. Development of a five-day basic microsurgery simulation training course: a cost analysis. Arch Plast Surg 2014;41:213-7.

35. Chang $\mathrm{CH}$. Medical simulation is needed in anesthesia training to achieve patient's safety. Korean J Anesthesiol 2013;64:204-11.

36. Stephenson LS, Gorsuch A, Hersh WR, et al. Participation in EHR based simulation improves recognition of patient safety issues. BMC Med Educ 2014;14:224.

37. Denadai R, Kirylko L. Teaching basic plastic surgical skills on an alternative synthetic bench model. Aesthet Surg J 2013;33:458-61.

38. Hamilton EC, Scott DJ, Kapoor A, et al. Improving operative performance using a laparoscopic hernia simulator. Am J Surg 2001;182:725-8.

39. Scott DJ, Bergen PC, Rege RV, et al. Laparoscopic training on bench models: better and more cost effective than operating room experience? J Am Coll Surg 2000;191:272-83.

40. Scott DJ, Dunnington GL. The new ACS/APDS skills curriculum: moving the learning curve out of the operating room. J Gastrointest Surg 2008;12:213-21.

41. Sturm LP, Windsor JA, Cosman PH, et al. A systematic review of skills transfer after surgical simulation training. Ann Surg 2008;248:166-79.

42. Cotin S, Delingette H, Ayache N. A hybrid elastic model allowing real-time cutting, deformations and force feedback for surgery training and simulation. Visual Comput 2000; $16: 437-52$

43. Vadodaria S, Watkin N, Thiessen F, et al. The first cleft palate simulator. Plast Reconstr Surg 2007;120:259-61.

44. Rosen JM, Long SA, McGrath DM, et al. Simulation in plastic surgery training and education: the path forward. Plast Reconstr Surg 2009; 123:729-38.

45. Palter VN, Grantcharov TP. Development and validation of a comprehensive curriculum to teach an advanced minimally invasive procedure: a randomized controlled trial. Ann Surg 2012;256:25-32.

46. Aboud E, Al-Mefty O, Yasargil MG. New laboratory model for neurosurgical training that simulates live surgery. J Neu- 
rosurg 2002;97:1367-72.

47. Aboud ET, Krisht AF, O'Keeffe T, et al. Novel simulation for training trauma surgeons. J Trauma 2011;71:1484-90.

48. Garrett HE Jr. A human cadaveric circulation model. J Vasc Surg 2001;33:1128-30.

49. Ericsson KA. Deliberate practice and the acquisition and maintenance of expert performance in medicine and related domains. Acad Med 2004;79(10 Suppl):S70-81.
50. Accreditation Council for Graduate Medical Education. The plastic surgery milestone project [Internet]. Chicago, IL: ACGME; c2015 [cited 2018 Feb 5]. Available from https://www.acgme.org/Portals/0/PDFs/Milestones/ PlasticSurgeryMilestones.pdf.

51. Gosman A, Mann K, Reid CM, et al. Implementing assessment methods in plastic surgery. Plast Reconstr Surg 2016; 137:617e-623e. 